\title{
Lack of integrase inhibitors associated resistance mutations among HIV-1C isolates
}

\author{
Andargachew Mulu ${ }^{1,2^{*}}$, Melanie Maier ${ }^{1}$ and Uwe Gerd Liebert ${ }^{1}$
}

\begin{abstract}
Background: Although biochemical analysis of HIV-1 integrase enzyme suggested the use of integrase inhibitors (INIs) against HIV-1C, different viral subtypes may favor different mutational pathways potentially leading to varying levels of drug resistance. Thus, the aim of this study was to search for the occurrence and natural evolution of integrase polymorphisms and/or resistance mutations in HIV-1C Ethiopian clinical isolates prior to the introduction of INIs.

Methods: Plasma samples from chronically infected drug naïve patients $(N=45)$, of whom the PR and RT sequence was determined previously, were used to generate population based sequences of HIV-1 integrase. HIV-1 subtype was determined using the REGA HIV-1 subtyping tool. Resistance mutations were interpreted according to the Stanford HIV drug resistance database (http://hivdb.stanford.edu) and the updated International Antiviral Society (IAS)-USA mutation lists. Moreover, rates of polymorphisms in the current isolates were compared with South African and global HIV-1C isolates.

Results: All subjects were infected with HIV-1C concordant to the protease (PR) and reverse transcriptase (RT) regions. Neither major resistance-associated IN mutations (T66I/A/K, E92Q/G, T97A, Y143HCR, S147G, Q148H/R/K, and $\mathrm{N} 155 \mathrm{H}$ ) nor silent mutations known to change the genetic barrier were observed. Moreover, the DDE-catalytic motif (D64G/D116G/E152 K) and signature HHCC zinc-binding motifs at codon 12, 16, 40 and 43 were found to be highly conserved. However, compared to other South African subtype $\mathrm{C}$ isolates, the rate of polymorphism was variable at various positions.
\end{abstract}

Conclusion: Although the sample size is small, the findings suggest that this drug class could be effective in Ethiopia and other southern African countries where HIV-1C is predominantly circulating. The data will contribute to define the importance of integrase polymorphism and to improve resistance interpretation algorithms in HIV-1C isolates.

Keywords: Integrase, Integrase inhibitors, HIV-1 subtype C, Drug resistance, Polymorphism/mutations, Ethiopia

\section{Background}

HIV replication requires three viral enzymes and accessory metabolites provided by the infected cell. The viral enzymes protease (PR), reverse transcriptase (RT), and Integrase (IN), encoded by the polymerase ( $p o l$ ) gene are the main targets of current antiretroviral drugs [1]. HIV-1 IN is a 288 amino acid (aa) protein encoded by the $5^{\prime}$ end of the pol gene that folds in a multimeric form into 3 functional domains: the N-terminal domain (NTD:

\footnotetext{
*Correspondence: andargachewmulu@yahoo.com; andargachewmulu. meharie@medizin.uni-leipzig.de

${ }^{1}$ Institute of Virology, Faculty of Medicine, University of Leipzig, Leipzig, Germany

Full list of author information is available at the end of the article
}

aa 1-49) contains an HHCC zinc binding motif which is essential to facilitate IN multimerization through its extensive contacts with adjacent catalytic core domain (CCD) monomers; the CCD (aa 50-212) contains the DDE motif of the catalytic triad D64, D116 and E152 and the viral DNA binding site; and the C-terminal domain (CTD: aa 213-288) has host DNA binding activity [2-5]. IN is responsible for chromosomal integration of the newly synthesized double strand viral DNA into the host genomic DNA [2]. This chromosomal integration is a multistep process grouped in 3 major steps. The first is the formation of the pre-integration complex (which allows entry of viral genomes into the cell nucleus). The second is $3^{\prime}$ processing which prepares both ends of the 
proviral DNA for integration. During this process, IN recognizes conserved sequences in the long terminal repeats promoting the removal of GT dinucleotide from the $3^{\prime}$ end, resulting in new $3^{\prime}$ hydroxyl ends [2]. This step occurs in the cytoplasm and involves the pre-integration complex, which consists of both viral and cellular proteins that help the pre-integration complex to migrate through nuclear pores [6]. The final step is strand transfer in which target DNA is cleaved and viral DNA is joined to the $5^{\prime}$ phosphate ends in the host chromosome which is most likely completed by the host DNA repair machinery [2]. These enable HIV-1 to establish a permanent genetic reservoir that can initiate new virus's production and to replicate through cellular mitosis $[4,5]$.

In industrialized countries integrase strand transfer inhibitors have been shown to lead to virological suppression in both treatment naïve patient as well as treatment-experienced individuals with multidrug-resistance to other drug classes [7]. However, drug resistance to this drug class has been shown to occur both in vivo and in vitro $[8,9]$. Currently, there are more than 40 substitutions specifically associated with the development of resistance to integrase inhibitors (INIs). Yet, the main mutational pathways associated with INIs resistance are limited to signature mutations at IN positions 66, 92, 143, 147,148 , and 155 [8-10]. The prevalence of INIs resistant viral strains has not yet been reported; although some studies have found that $95 \%$ of HIV-1B-infected patients treated with this drug class were susceptible and showed viral suppression [7-9]. Even though biochemical analysis of HIV-1B and C integrase enzymes suggested the use of INIs against HIV-1C, recent studies have indicated that different viral subtypes may favor different mutational pathways potentially leading to varying levels of drug resistance among different subtypes and within the same subtype in different regions [11-13]. On top of that, IN sequence data on HIV-1C which is the most prevalent circulating clade in sub-Saharan African countries is lacking [12, 13]. So, it is worth enough to conduct specific studies on the HIV-1C subtype. Besides, an increasing number of patients in sub-Saharan African countries require alternative regimes as they fail first and second line regimes containing non-nucleoside reverse transcriptase inhibitors (NNRTIs), nucleoside reverse transcriptase inhibitors (NRTIs) and protease inhibitors (PIs) due to transmitted or secondary drug resistance mutations [14-20]. This is in line with our findings of a high rate of mutations conferring resistance to NNRTIs and NRTIs inhibitors in treatment naïve $[19,20]$ and treated Ethiopian patients [21]. Thus, the aim of this study was to search for the occurrence and natural evolution of integrase polymorphisms and/or integrase inhibitors (INIs) resistance mutations in HIV-1C clinical isolates of ART naïve Ethiopian patients prior to the introduction of INIs of whom the PR and RT sequence was determined previously [19].

\section{Methods}

\section{Patients and samples}

HIV-1C chronically infected treatment naïve patients above 18 years of age and seeking treatment at Gondar University Hospital, Northwest Ethiopia in 2008 were recruited consecutively. The inclusion and exclusion criteria and the baseline characteristics of the patients and sample collection were described before [20]. Briefly, five $\mathrm{ml}$ venous blood was collected in vacutainer tubes containing ethylene diamine tetraacetic acid. Baseline $\mathrm{CD} 4^{+}$ $\mathrm{T}$ cell count was measured using the FACSCount flow cytometer (Becton-Dickinson, San Jose, CA, USA) following the manufacturer's protocol. Plasma samples were separated by centrifugation and stored at $-40{ }^{\circ} \mathrm{C}$. RNA extraction and plasma viral load determination was made with the Abbott m2000sp automated sample preparation system using mSample preparation system RNA kit and Abbott m2000rt using Quantitative Realtime HIV-1 assay, respectively (Abbott Molecular, Des Plaines, IL, USA). The lower detection limit of the assay is 40 copies/ $\mathrm{ml}$.

\section{Amplification and sequencing}

The IN region of the pol gene of the HIV-1 genome was amplified by using an in-house protocol. RNA elute was reverse transcribed to cDNA using AMV reverse transcriptase (Promega Corporation, WI, USA) by an outer primer HIVINT-Rev1 (5'TGGGATGTGTACTTCTGA ACTTA3' corresponding to positions 5192-5214) at $50{ }^{\circ} \mathrm{C}$ for $1 \mathrm{~h}$. Viral cDNA was amplified by nested PCR using Phusion Hot Start High-Fidelity DNA polymerase (Finnzymes, Espoo, Finland) by outer primers HIVINTFor1 (5'AAAGGAATTGGAGGAAATGAAC3' corresponding to positions 4167-4188) and HIVINT-Rev1 and inner primers HIVINT-For2 (5'GAAATGAACAAGTAGATAAATTAGTAAG3' corresponding to positions 4180-4204) and HIVINT-Rev2 (5'CCTGCCATCTG TTTTCCATA3' corresponding to position 5040-5059). Initial denaturation was done at $98{ }^{\circ} \mathrm{C}$ for $2 \mathrm{~min}$ followed by 40 cycles consisting of $10 \mathrm{~s}$ of denaturation at $98{ }^{\circ} \mathrm{C}$ and $25 \mathrm{~s}$ of annealing at $64{ }^{\circ} \mathrm{C}$ for the first round and at $58^{\circ} \mathrm{C}$ for the second round with a $40 \mathrm{~s}$ extension at $72{ }^{\circ} \mathrm{C}$ for both and final extension for $5 \mathrm{~min}$ at $72{ }^{\circ} \mathrm{C}$. All positions are matched to HIV-1 HXB2 (GenBank accession number K03455). The RT-PCR products which showed a clear band on agarose gel were cut-off and purified by Wizard Promega PCR clean-up system (Promega, 
Madison, WI, USA) according to the manufacturer's instructions.

Purified PCR products (PCR Clean-up System, Promega) were subjected to direct sequencing of both the sense and antisense strands using Big Dye Terminator Cycle Sequencing Ready Reaction kit (Applied Biosystems Incorporated, Foster City, CA, USA). Sequencing was performed using the two inner primers which allowed a double coverage of the IN genome. After running the sequencing reaction, non-incorporated dideoxynucleoside triphosphates were removed by ethanol-sodium acetate precipitation and dried by vacuum centrifugation for $12 \mathrm{~min}$. The pellet was re-suspended in $20 \mu \mathrm{l}$ high density formamide (Applied Biosystems Incorporated, Foster City, CA, USA) for denaturation and loading on the ABI prism 310 Genetic Analyzer (Applied Biosystems). Both forward and reverse overlapping sequences were manually edited with the Geneious Basic software version 5.4 and exported as a FASTA format consensus sequences. Sequences were aligned with reference set from the Los Alamos HIV database (http://www. hiv.lanl.gov) using the Mega version 5 software (http:// www.megasoftware.net) and phylogenetic inferences were performed by the Neighbour-Joining (NJ) method under Kimura's two-parameter correction. One thousand bootstrap replicates were used to assess the phylogenetic robustness of the clusters. Moreover, the REGA HIV-1 subtyping tool was used for each sequence to determine the HIV-1 subtype.

\section{Drug resistance analysis}

Drug resistance mutations were analysed using The Stanford University HIV drug resistance database (http://hivdb.stanford.edu) and the 2015 updated drug resistance mutations list of the International Antiviral Society (IAS-USA) [22]. Although, frequency cut-off to distinguish polymorphic from non-polymorphic positions has not been proposed amino acid changes with a prevalence of at least $3 \%$ among treatment-naive patients were considered, as used previously [23]. The rates of polymorphisms in the current study were also compared with South African subtype C $(\mathrm{N}=72)$ specific polymorphisms reported by Fish et al. [12] and with the Global HIV-1 subtype C $(\mathrm{N}=1044)$ isolates (http:// hivdb.stanford.edu).

\section{Statistical analyses}

The data was analysed using to SPSS version 17 statistical packages. HIV-RNA loads were transformed to $\log _{10}$ for analysis. Data were summarized as medians and interquartile range (IQR). Non-parametric tests were performed to compare median $\mathrm{CD} 4^{+} \mathrm{T}$ cells count and plasma HIV-RNA levels of the different groups.

\section{Ethical approval}

The study protocol and design including the consent procedures were approved by the University of Gondar Ethical Review Committee (RPO/55/291/00). Patients were managed following the national guideline. Written informed consent was obtained from all study subjects and documented in Research Office of the University.

\section{Results}

From the total of 45 patients enrolled in this study, $53 \%$ were females. The mean $\pm \mathrm{SD}$ age of the subjects was $33 \pm 1.6$ years ranging from 24 to 58 years. There was no significant difference in the mean age among males and females (32.4 versus 34.5, respectively; $\mathrm{P}=0.086$ ). According to the WHO AIDS stage defining criteria, $15.6 \%$ (7/45), $20 \%$ (9/45), $55.6 \%(25 / 45)$ and $8.8 \%(4 / 45)$ of the patients were classified into stage one, two, three and four, respectively. Baseline median $\mathrm{CD} 4^{+} \mathrm{T}$ cell count was 103 cells $/ \mathrm{mm}^{3}$ (IQR: $57.0-287.0$ ) and the proportion of patients with CD4 ${ }^{+} \mathrm{T}$ cell count $\leq 200$ and $>201$ cells $/ \mathrm{mm}^{3}$ was 62 and $38 \%$, respectively. The mean $\log _{10}$ HIV-1 RNA level of the patients was 4.54 and did not significantly differ among patients with higher and lower CD4 ${ }^{+} \mathrm{T}$ cells strata $\left(\log _{10} 4.65\right.$ for $>201$ cells $/ \mathrm{mm}^{3}$ versus $\log _{10} 4.4$ for $\leq 200$ cells $/ \mathrm{mm}^{3}$ ). As previously reported [20] in this group of well characterized patients, two patients had NNRTI associated mutations (G190A and E138G) and one patient had a NRTI resistance-associated mutation (L210 W). A subtype C-specific polymorphism associated with NRTIs (V118I) was detected in one patient. No major drug resistance mutations in PR region were found (Table 1). However, the presence of subtype $C$ specific polymorphisms and minor resistance mutations to protease inhibitors were detected frequently.

The 45 nearly full-length nucleotide sequences of the IN region covering the first 269 amino acids (93\% of the IN gene) were with intact with open reading frames and without frameshift deletion or insertion confirming the presence of functional IN genes in all patients and primary virus isolates. However, a deletion of 6 and 12 amino acids in the catalytic core domain-CCD from codon 122 to codon 127 and from codon 188 to codon 199 was observed in two of the samples (5550 and 5713) obtained from a 43 years old male and a 38 years old female patient with baseline $\log _{10}$ HIV RNA level of 4.66 and 3.86 and $C D 4^{+}$T cell count of 209 and 182 cells $/ \mathrm{mm}^{3}$, respectively. Such a massive deletion has not been observed in several HIV-1C and other sequences available in HIV database. The IN intra-subtype nucleotide diversity varied between $1.7 \%$ and $5.4 \%$ which is similar to what has been reported previously from South African subtype C IN sequences [12, 13]. All sequences were found to be HIV-1C in concordance with the 
Table 1 HIV-1 genotype drug resistance profile to PRI, RTI and INIs of chronically infected HIV-1 subtype C patients

\begin{tabular}{|c|c|c|c|c|c|c|}
\hline ID & Age/sex & RNA & CD4 count & Resistance to PRIs & Resistance to RTIs & Resistance to INIs \\
\hline 5572 & $28 / \mathrm{M}$ & 5.18 & 20 & Minor R (V11FV) & S & S \\
\hline 5492 & $30 / F$ & 5.38 & 27 & S & S & S \\
\hline 5493 & $23 / F$ & 5.28 & 33 & S & S & S \\
\hline 5790 & $45 / M$ & 4.18 & 37 & S & S & S \\
\hline 5531 & $24 / F$ & 6.17 & 38 & S & S & S \\
\hline 5655 & $29 / F$ & 3.75 & 48 & S & S & S \\
\hline 5551 & $28 / M$ & 4.66 & 59 & $S$ & S & S \\
\hline 5642 & $25 / F$ & 4.01 & 60 & S & S & S \\
\hline 5775 & $45 / F$ & 3.85 & 61 & S & S & S \\
\hline 5595 & $25 / M$ & 4.32 & 66 & S & S & S \\
\hline 5592 & $55 / M$ & 5.82 & 75 & S & S & S \\
\hline 5717 & $28 / F$ & 4.21 & 75 & S & S & S \\
\hline 5525 & $30 / F$ & 4.79 & 80 & S & S & S \\
\hline 5648 & $35 / F$ & 5.04 & 85 & S & S & S \\
\hline 5591 & $33 / M$ & 4.37 & 85 & S & S & S \\
\hline 5582 & $32 / \mathrm{M}$ & 5.43 & 89 & S & S & S \\
\hline 5568 & $36 / M$ & 3.90 & 92 & S & S & S \\
\hline 5499 & $35 / M$ & 5.91 & 97 & S & S & S \\
\hline 5604 & $27 / M$ & 4.64 & 97 & S & S & S \\
\hline 5517 & $25 / F$ & 3.83 & 112 & S & S & S \\
\hline 5627 & $30 / F$ & 3.08 & 115 & S & S & S \\
\hline 5732 & $36 / F$ & 5.14 & 118 & S & S & S \\
\hline 5479 & $35 / F$ & 5.44 & 124 & S & R-NNRTIs (E138G) & S \\
\hline 5546 & $38 / F$ & 4.33 & 125 & S & S & S \\
\hline 5841 & $58 / \mathrm{M}$ & 4.47 & 129 & S & S & S \\
\hline 5733 & $32 / F$ & 4.78 & 146 & S & S & S \\
\hline 5713 & $38 / F$ & 3.86 & 182 & S & S & S \\
\hline 5681 & $28 / \mathrm{M}$ & 4.38 & 191 & S & S & S \\
\hline 5550 & $43 / M$ & 4.66 & 209 & S & S & S \\
\hline 5786 & $27 / F$ & 3.88 & 211 & S & S & S \\
\hline 5837 & $28 / F$ & 3.76 & 212 & S & S & S \\
\hline 5768 & $44 / M$ & 5.91 & 219 & S & S & S \\
\hline 5712 & $39 / M$ & 4.82 & 224 & S & R-NNRTIs (G190A) & S \\
\hline 5669 & $40 / F$ & 3.57 & 227 & Minor R (L10I) & S & S \\
\hline 5585 & $47 / F$ & 4.60 & 227 & S & S & S \\
\hline 5596 & $27 / F$ & 4.8 & 238 & S & S & S \\
\hline 5511 & $20 / F$ & 4.54 & 262 & S & S & S \\
\hline 5524 & $42 / M$ & 3.90 & 269 & S & S & S \\
\hline 5485 & $40 / \mathrm{M}$ & 4.50 & 277 & S & S & S \\
\hline 5690 & $30 / \mathrm{M}$ & 3.30 & 312 & S & S & S \\
\hline 5530 & $24 / F$ & 3.81 & 336 & S & S & S \\
\hline 5582 & $34 / M$ & 5.43 & 366 & S & S & S \\
\hline 5630 & $28 / \mathrm{M}$ & 4.17 & 401 & S & S & S \\
\hline 5511 & $24 / F$ & 4.54 & 421 & S & S & S \\
\hline 5496 & $40 / \mathrm{M}$ & 3.88 & 751 & S & R-NRTIs (L210 W) & S \\
\hline
\end{tabular}

$M$ Male, $F$ Female, HIV RNA in $\log _{10}$ copies $/ \mathrm{ml}$; CD4 ${ }^{+} \mathrm{T}$ cell count (cells $/ \mathrm{mm}^{3}$ ); PRI protease inhibitors, $R T /$ reverse transcriptase inhibitors, INI integrase inhibitors, $S$ susceptible, $R$ resistance 
corresponding PR and RT sequences [19] which is additional evidence for clade homogeneity in the region. Phylogenetic analysis of the integrase nucleotide sequences, together with viruses from the major subtypes, revealed that the majority of sequences clustered with Ethiopian HIV-1C isolates which indicates that the majority of the current Ethiopian HIV-1 epidemic descended from a single introduction into the country (Fig. 1). Clear cluster of sequences with sequences of other countries (Brazil, S. African and Indian and China) was also observed indicating that HIV-1 subtype sequences from these countries are inter-related and/or subtype $\mathrm{C}$ in Ethiopia may have been introduced from these countries HIV-1C lineages. None of the isolates revealed recombination.

Table 2 shows the frequency of the IN sequences with mutations and/or polymorphisms associated with reduced susceptibility to INIs. As expected, none of the samples contained mutations associated with primary resistance to INIs and all the isolates were found to be susceptible for INIs (Tables 1, 2). Moreover, the DDE-catalytic motif (D64G/D116G/E152 K) and signature HHCC zinc-binding motifs at codon 12, 16, 40 and 43 were found to be highly conserved. Silent mutations leading to higher increment of genetic barrier were not detected in the current study. However, polymorphic and non-polymorphic changes were observed (Table 2). Three patients (6.7 \%) had minor INI-resistance mutations, i.e. L74 M and T97A. Unusual mutations at minor INI-resistance positions were observed in small number of patients (Table 2). Two polymorphic minor INI-accessory mutations (V201I and I203 M) occurred in 82.2 and $13.3 \%$ of the patients, respectively. The rates of polymorphisms in

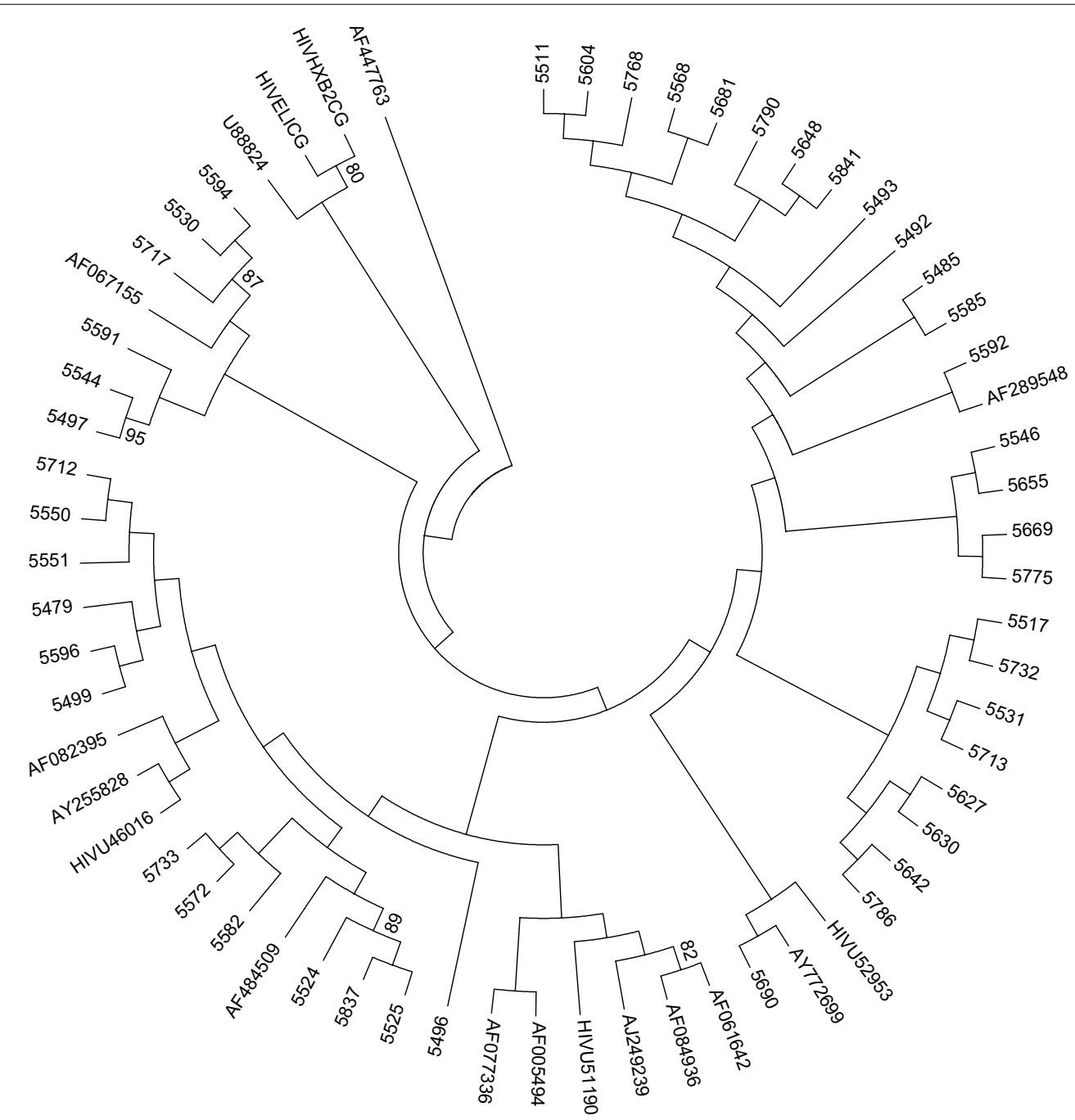

Fig. 1 Phylogenetic relationships of the IN HIV-1C sequences (4 digit number-GenBank Accession number: KF959731-KF959775) with different HIV-1 subtype reference sequences from the Los Alamos database (http://hiv-web.lanl.gov). Bootstrap values greater than $70 \%$ are indicated 
Table 2 Pattern and frequency mutations and polymorphisms with potential impact on INIs reduced susceptibility of INIs among HIV-1 subtype $C(n=45)$

\begin{tabular}{lc}
\hline Minor INI-resistance mutations & Frequencey \\
\hline L74 M & $1(2.2)$ \\
L74I & $4(8.8)$ \\
T97A & $2(4.4)$ \\
E138X & $4(8.8)$ \\
G163E & $6(13.3)$ \\
G163 Va & $2(4.4)$ \\
G163 Wa & $3(6.6)$ \\
Minor INI accessory mutations & \\
V201I & $37(82.2)$ \\
I203 M & $6(13.3)$ \\
\hline
\end{tabular}

a Unusual mutations in non-polymorphic sites associated with minor INI mutations (according to The Stanford University HIV drug resistance database; http://hivdb.stanford.edu)

the current study compared with South African HIV-1C specific polymorphisms [12] were similar at 6 positions, but significantly higher at $8 / 21$ positions and significantly lower at $7 / 21$ positions (Fig. 2).

\section{Discussion}

In this study, the IN aa sequence alignment was screened for the presence of major mutations, non-polymorphic and polymorphic changes associated with resistance to INIs (http://hivdb.stanford.edu) and additionally was categorized according to a previous report associated with INI resistance [23] (1) Major INI-resistance mutations were defined as mutations that phenotypically decrease susceptibility to raltegravir or elvitegravir by fivefold or higher and have been reported to be selected by one of these INIs in vitro or in vivo. This includes T66IAK, E92Q, F121Y, G140SA, Y143HCR, Q146P, S147G, Q148KHR, and N155HS; (2) minor INI-resistance mutations were defined as non-polymorphic or minimally polymorphic mutations that reduce INI susceptibility < fivefold by themselves or that significantly contribute to resistance when they occur in combination with other mutations (H51Y, L74 M, T97A, E138AK, S153Y, E157Q, G163RK, S230R, and R263 K); and (3) minor INI accessory mutations were defined as highly polymorphic mutations that have been reported to occur more frequently among INI-treated than INI naive patients but which have not been shown to contribute to reduced INI susceptibility (V68VI, V151IA, M154IL, V201I, I203 M, and $\mathrm{S} 230 \mathrm{~N}$ ).

The present study describes for the first time the occurrence of natural genetic polymorphisms in the IN region among HIV-1C isolates from the Horn of Africa and identifies the frequency of natural polymorphisms associated with resistance to INIs in samples from chronically infected antiretroviral drug-naïve patients. The absence of mutations associated with primary resistance to INIs and silent mutations (e.g. at codon 151) leading to higher increment of genetic barrier [24] is as expected and similar with previous reports from South Africa [11, 12], Mozambique [25], Cameron [26], Brazil [27], Quebec [28] and other parts of the world [23]. The occurrences of polymorphic minor INI-accessory mutations (L74I,

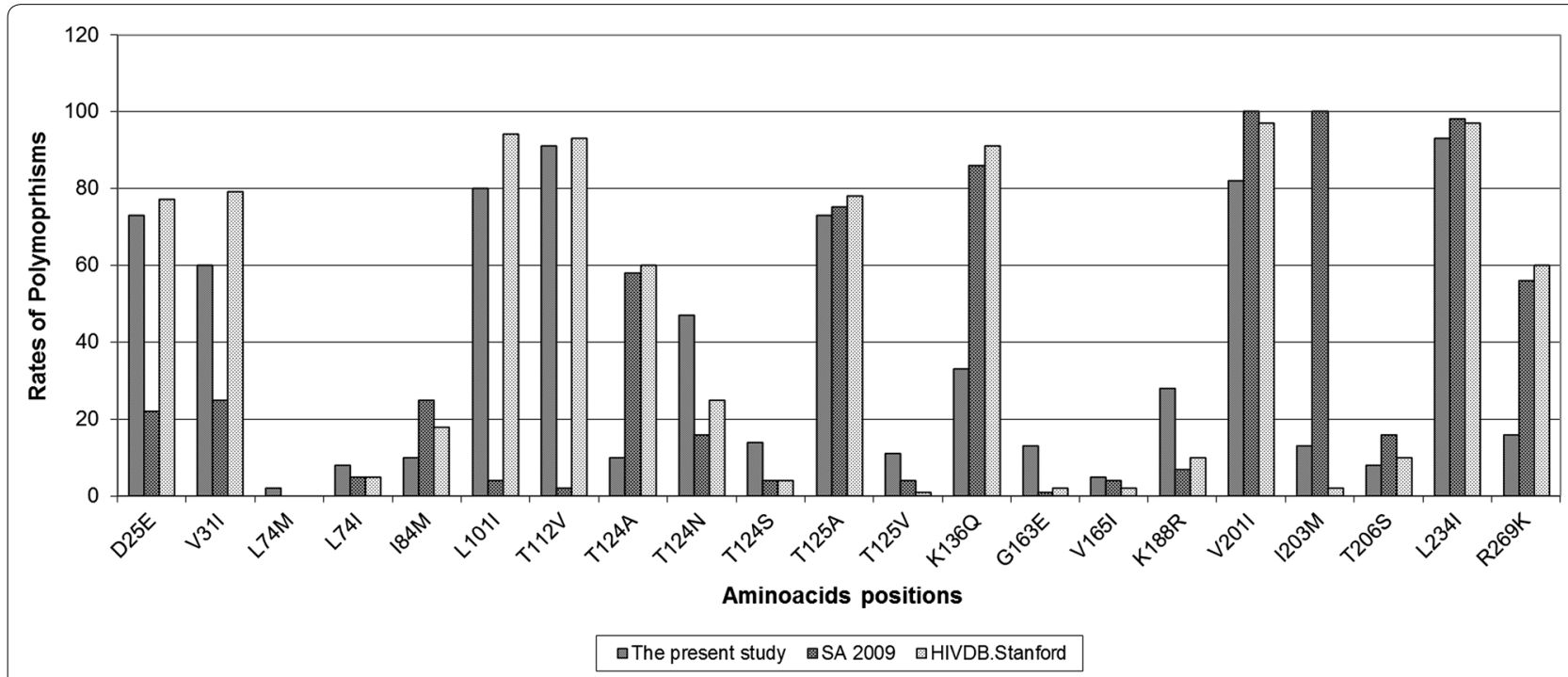

Fig. 2 Distribution of HIV-1C IN sequences polymorphic and non-polymorphic changes of the present study (ETH 2008/2009) compared with the South African HIV-1C (SA 2009) [12] and global HIV-1C sequences (http://hivdb.stanford.edu) 
T125A, V165I, T206S, L234I and V201I) are similar to previous HIV-1C isolates from South Africa [11, 12]. However, the variations on the rates of polymorphisms observed in the current study compared with South African subtype $C$ specific polymorphisms [12] (Fig. 2) suggest the presence of intra-subtype region-specific differences.

The current study shows regional specific polymorphic and non-polymorphic changes as reported previously from southern Africa, Brazil and Quebec [28]. The absence of the non-polymorphic minor INI-resistance mutations (E157Q which could be selected by raltegravir reducing elvitegravir susceptibility) in the current study is similar with a recent report from Brazilian subtype C isolates [27] and several other studies [23, 29]. But, it was inconsistent with the findings from Quebec [28] and from South Africa $[11,12]$ reported in 35 and $4.1 \%$ of the isolates, respectively suggesting subtype $C$ regional variations involving epidemics from Ethiopia, South Africa and Quebec. On the other hand, a highly polymorphic minor INI accessory mutation (V201I) was detected in $82 \%$ of the isolates which is significantly higher compared with the report from Cameron [26] and Quebec [28] subtype $C$ isolates and consistent with South African isolates [11, 12]. These findings may explain the differing rates of acquisition and accumulation of mutations in subtype $C$ isolates [30-32]. Although the significance of these residues to the current generation of INIs is not yet well known, the current findings show the need for surveillance of IN mutations and HIV-1C region specific phenotypic studies to explain the relevance of the naturally occurring polymorphisms in the absence of the signature IN mutations on susceptibility/ resistance to INIs.

\section{Conclusion}

None of the previously reported major mutations (T66AIK, E92Q, Y143RCH, S147G, Q148HRK and $\mathrm{N} 155 \mathrm{H}$ ) associated with resistance to INIs were observed in HIV-1C Ethiopian isolates, indicating that INIs can be used as a treatment option in Ethiopia and other African countries where HIV-1C is predominately circulating. However, the IN sequence variations found in the present study compared with other African subtype $\mathrm{C}$ isolates needs further investigation. The findings of the present study may be useful not only to explain natural IN genetic polymorphism but also to improve resistance interpretation algorithms in HIV-1C isolates about which very little is known.

Sequence data: Nucleotide sequences are deposited in National Centre for Biotechnology Information (NCBI), USA with Accession number [GenBank: KF959731-KF959775].

\section{Authors' contributions}

AM: Conception and design of the study, acquisition, analysis and interpretation of data, drafting the article and final approval of the version to be submitted; MM: Conception and design of the study, analysis and interpretation of data, revising the draft article and final approval of the version to be submitted; UGL: Conception and design of the study, analysis and interpretation of data, revising the draft article and final approval of the version to be submitted. All authors read and approved the final manuscript.

\section{Author details}

${ }^{1}$ Institute of Virology, Faculty of Medicine, University of Leipzig, Leipzig, Germany. ${ }^{2}$ Department of Microbiology, College of Medicine and Health Sciences, University of Gondar, Gondar, Ethiopia.

\section{Acknowledgements}

The authors would like to thank all study participants. Expert technical assistance by Sandra Bergs and Janka Rätzke is gratefully acknowledged. This work was partly supported by German Academic Exchange Service (DAAD), Association of Sponsors and Friends of Leipzig University, and HIV/AIDS Prevention and Control Office of Amhara Regional State, Ethiopia. The funders had no any role in study design, data collection and analysis, decision to publish, or preparation of the manuscript.

\section{Competing interests}

The authors declare that they have no competing interests.

Received: 15 July 2015 Accepted: 18 November 2015

Published online: 01 December 2015

\section{References}

1. Sarafianos SG, Marchand B, Das K, Himmel DM, Parniak MA, Hughes $\mathrm{SH}$, et al. Structure and function of HIV-1 reverse transcriptase: molecular mechanisms of polymerization and inhibition. J Mol Biol. 2009;385:693-713.

2. Engelman A, Krishnan L. Retroviral integrase proteins and HIV-1 DNA integration. J Biol Chem. 2012;287:40858-66. doi:10.1074/jbc.R112.397760.

3. Van Maele B, Busschots K, Vandekerckhove L, Christ F, Debyser Z. Cellular co-factors of HIV-1 integration. Trends Biochem Sci. 2006;31:98-105.

4. Chiu TK, Davies DR. Structure and function of HIV-1 integrase. Curr Top Med Chem. 2004:4:965-77.

5. Craigie R. HIV integrase, a brief overview from chemistry to therapeutics. J Biol Chem. 2000;276:23213-6.

6. Van Maele B, Debyser Z. HIV-1 integration: an-interplay between HIV-1 integrase, cellular and viral proteins. AIDS Rev. 2005;7:26-43.

7. Cooper DA, Steigbigel RT, Gatell JM, Rockstroh JK, Katlama C, Yeni P, et al. Subgroup and resistance analyses of raltegravir for resistant HIV-1 infection. N Engl J Med. 2008;359:355-65.

8. Buzón MJ, Marfil S, Puertas MC, Garcia E, Clotet B, Ruiz L, et al. Raltegravir susceptibility and fitness progression of HIV type-1 integrase in patients on long-term antiretroviral therapy. Antivir Ther. 2008;13:881-93.

9. Charpentier C, Laureillard D, Piketty C, Tisserand P, Batisse D, Karmochkine $M$, et al. High frequency of integrase Q148R minority variants in HIVinfected patient's naive of integrase inhibitors. AIDS. 2010;24:867-73.

10. Quercia R, Dam E, Perez-Bercoff D, Clavel F. Selective advantage profile of human immunodeficiency virus type 1 integrase mutants explains in vivo evolution of raltegravir resistance genotypes. J Virol. 2009;83:10245-9.

11. Bar-Magen T, Donahue DA, McDonough El, Kuhl BD, Faltenbacher VH, $\mathrm{Xu} \mathrm{H}$, et al. HIV-1 subtype $\mathrm{B}$ and $\mathrm{C}$ integrase enzymes exhibit differential patterns of resistance to integrase inhibitors in biochemical assays. AIDS. 2010;24:2171-9.

12. Fish MQ, Hewer R, Wallis CL, Venter WD, Stevens WS, Papathanasopoulos MA. Natural polymorphisms of integrase among HIV type 1-infected South African patients. AIDS Res Hum Retrovir. 2010;26:489-93. doi:10.1089/aid.2009.0249.

13. Papathanasopoulos MA, Vardas E, Wallis C, Glashoff R, Buttó S, Poli G, et al. Characterization of HIV type 1 genetic diversity among South African participants enrolled in the AIDS vaccine integrated project (AVIP) study. AIDS Res Hum Retrovir. 2010;26:705-9. doi:10.1089/aid.2009.0281. 
14. Hosseinipour MC, van Oosterhout JJ, Weigel R, Phiri S, Kamwendo D, Parkin $\mathrm{N}$, et al. The public health approach to identify antiretroviral therapy failure: high-level nucleoside reverse transcriptase inhibitor resistance among Malawians failing first-line antiretroviral therapy. AIDS. 2009;23:1127-34

15. Sigaloff KC, Hamers RL, Wallis CL, Kityo C, Siwale M, Ive P, et al. Unnecessary antiretroviral treatment switches and accumulation of HIV resistance mutations; two arguments for viral load monitoring in Africa. J Acquir Immune Defic Syndr. 2011;58:23-31.

16. Wallis CL, Mellors JW, Venter WD, Sanne I, Stevens W. Varied patterns of HIV-1 drug resistance on failing first-line antiretroviral therapy in South Africa. J Acquir Immune Defic Syndr. 2010;53:480-4.

17. Koyalta D, Charpentier C, Beassamda J, Rey E, Si-Mohamed A, DjemadjiOudjeil N, et al. High frequency of antiretroviral drug resistance among HIV-infected adults receiving first-line highly active antiretroviral therapy in N'Djamena, Chad. Clin Infect Dis. 2009;49:155-9.

18. Marconi VC, Sunpath H, Lu Z, Gordon M, Koranteng-Apeagyei K, Hampton J, et al. Prevalence of HIV-1 drug resistance after failure of a first highly active antiretroviral therapy regimen in Kwa Zulu Natal, South Africa. Clin Infect Dis. 2008;46:1589-97.

19. Mulu, Lange T, Liebert UG, Maier M. Clade homogeneity and Pol gene polymorphisms in chronically HIV-1 infected antiretroviral treatment naive patients after the roll out of ART in Ethiopia. BMC Infect Dis. 2014;. doi:10.1186/1471-2334-14-158.

20. Huruy K, Maier M, Mulu A, Liebert UG. Limited increase in primary HIV-1C drug resistance mutations in treatment naïve individuals in Ethiopia. J Med Virol. 2015;87(6):978-84. doi:10.1002/jmv.24110.

21. Mulu A, Maier M, Liebert UG. Low incidence of HIV-1C acquired drug resistance 10 years after roll-out of antiretroviral therapy in Ethiopia: a prospective cohort study. PLoS One. 2015;10(10):e0141318. doi:10.1371/ journal.pone.0141318.

22. Wensing AM, Calvez V, Günthard HF, Johnson VA, Paredes R, Pillay D, et al. Update of the drug resistance mutations in HIV-1: international AIDS society-USA. Top Antivir Med. 2015; 23(4) (Epub ahead of print).
23. Rhee SY, Liu TF, Kiuchi M, Zioni R, Gifford RJ, Holmes SP, et al. Natural variation of HIV-1 group M integrase: implications for a new class of antiretroviral inhibitors. Retrovirology. 2008;5:7. doi:10.1186/1742-4690-5-74.

24. Steigbigel RT, Cooper DA, Kumar PN, Eron JE, Schechter M, Markowitz M, et al. Raltegravir with optimized background therapy for resistant HIV-1 infection. N Engl J Med. 2008;359:339-54

25. Oliveira MF, Ramalho DB, Abreu CM, Vubil A, Mabunda N, Ismael N, et al. Genetic diversity and naturally polymorphisms in HIV type 1 integrase isolates from Maputo, Mozambique: implications for integrase inhibitors. AIDS Res Hum Retrovir. 2012;28:1788-92. doi:10.1089/aid.2012.0058.

26. Turriziani O, Montagna C, Falasca F. Analysis of the integrase gene from hiv-1 positive patients living in a rural area of west Cameroon. AIDS Res Hum Retrovir. 2012;28:1729-33. doi:10.1089/AID.2011.0266.

27. Passaes CB, Guimarães ML, Fernandez SL, Lorete Rdos S, Teixeira SL, Fernandez JC, et al. Lack of primary mutations associated with integrase inhibitors among HIV-1 subtypes B, C, and F circulating in Brazil. J Acquir Immune Defic Syndr. 2000;51:7-12.

28. Brenner BG, Lowe M, Moisi D, Hardy I, Gagnon S, Charest H, et al. Subtype diversity associated with the development of HIV-1 resistance to integrase inhibitors. J Med Virol. 2011;83:751-9.

29. Eshleman SH, Hudelson SE, Smith P, Hackett J, Holzmayer V, Swanson $\mathrm{P}$, et al. Analysis of pol integrase sequences in diverse HIV type 1 strains using a prototype genotyping assay. AIDS Res Hum Retrovir. 2009;25:343-5. doi:10.1089/aid.2008.0236.

30. Abebe A, Pollakis G, Fontanet AL, Fisseha B, Tegbaru B, Kliphuis A, et al. Identification of a genetic subcluster of HIV type 1 subtype $C\left(C^{\prime}\right)$ widespread in Ethiopia. AIDS Res Hum Retrovir. 2000;16:1909-14.

31. Pollakis G, Abebe A, Kliphuis A, De Wit TF, Fisseha B, Tegbaru B, et al. Recombination of HIV type $1 C$ ( $\left.C^{\prime} / C\right)$ in Ethiopia: possible link of EthHIV$1 C^{\prime}$ to subtype $\mathrm{C}$ sequences from the high-prevalence epidemics in India and Southern Africa. AIDS Res Hum Retrovir. 2003;19:999-1008.

32. Soares EA, Santos AF, Sousa TM, Sprinz E, Martinez AM, Silveira J, et al. Differential drug resistance acquisition in HIV-1 of subtypes B and C. PLoS One. 2007;15(28):730.

\section{Submit your next manuscript to BioMed Central and we will help you at every step:}

- We accept pre-submission inquiries

- Our selector tool helps you to find the most relevant journal

- We provide round the clock customer support

- Convenient online submission

- Thorough peer review

- Inclusion in PubMed and all major indexing services

- Maximum visibility for your research

Submit your manuscript at www.biomedcentral.com/submit

\section{() Biomed Central}

